\title{
CARACTERIZAÇÃO DAS PROPRIEDADES FÍSICAS DE ESPÉCIES TROPICAIS COMERCIALIZADAS POR MATO GROSSO ${ }^{1}$
}

\author{
Edilene Silva Ribeiro ${ }^{2}$ \\ Joaquim Carlos Gonçalez ${ }^{3}$ \\ Luzia Elaine Domingues Pimenta Vargas ${ }^{2}$
}

\section{INTRODUÇÃO}

As folhosas de maneira geral possuem um sistema biológico complexo e variável acarretando alterações nas propriedades anatômicas, químicas, físicas e mecânicas entre espécies, entre indivíduos de uma mesma espécie e entre diferentes partes de um mesmo indivíduo (OLIVEIRA e SILVA, 2003). Diversas pesquisas têm sido desenvolvidas com o intuito de verificar os fatores que afetam essas propriedades, que podem ser intrínsecos à própria madeira ou de sítio, fertilidade, microfauna, clima e ambiente (BAKER et al., 2004; LOBÃO et al., 2004).

De acordo com Moreschi (2010), a densidade é um parâmetro muito utilizado na classificação e qualidade da madeira. Entre as várias propriedades, a densidade é a mais relevante, pois além de ser mensurada com facilidade, tem alta correlação com a massa, composição celular, demais propriedades físicas, mecânicas, térmicas, acústicas e elétricas (BATISTA, KLITZKE E SANTOS, 2010; BATISTA, 2012). Em espécies tropicais estas variações são decorrentes da anatomia de cada indivíduo, a exemplo: quantidade e volume dos vasos e parênquimas, espessura da parede celular, dimensão das fibras e em proporção menor a quantidade de extrativos (OLIVEIRA eSILVA, 2003).

1 Parte da tese de doutorado da primeira autora submetida ao Programa de Pós-Graduação em Ciências Florestais do Departamento de Engenharia Florestal da Universidade de Brasília (PPGEFL/UnB).

2 Professora do Ensino Básico, Técnico e Tecnológico do Instituto Federal de Mato Grosso (IFMT), Campus Campo Novo do Parecis. E-mails. Edilene.ribeiro@cnp.ifmt.edu.br; luzia.pimenta@cnp.ifmt.edu.br

3 Professor no Programa de Pós-Graduação em Ciências Florestais, Departamento de Engenharia Florestal, Faculdade de tecnologia, Universidade de Brasília (UnB). E-mail. goncalez@unb.br 
Já a umidade apesar de não ser uma característica intrínseca da madeira, seu estudo torna-se significativo por afetar o comportamento do material quanto à instabilidade dimensional, resistência mecânica e durabilidade. Por ser um material altamente higroscópico, suas variações dimensionais geradas pela contração e inchamento associados à anisotropia, constituem características indesejáveis, limitando seu uso, ou ainda, exigindo técnicas mais específicas para a utilização da madeira (DURLO e MARCHIORI, 1992; OLIVEIRA E SILVA, 2003).

A retratibilidade da madeira é definida como a variação nas suas dimensões quando há alteração teor de umidade, resultando na movimentação pela perda ou ganho de água abaixo do ponto de saturação das fibras (OLIVEIRA, 1998; MARQUES et al., 2012). Ocorre pelo fato das moléculas de água estarem ligadas por pontes de hidrogênio às microfibrilas dos polissacarídeos que formam a madeira, de forma que quando estas são forçadas a sair, deixam um vazio. As forças de coesão existentes então tendem a reaproximar essas microfibrilas, causando a contração da madeira. $O$ evento da expansão é o inverso, ou seja, quando a água é adsorvida pela madeira tende a penetrar entre as microfibrilas e causa o seu afastamento e, como consequência, o inchamento da peça de madeira OLIVEIRA et al., 2010).

O coeficiente de anisotropia é a razão entre as contrações radial e tangencial (T/R) e representam o comportamento da madeira em relação à secagem. Durlo e Machiori, (1992) discorrem que uma madeira com fator anisotrópico 1 representa igualdade de contração das faces radial e tangencial, ou seja, madeira estável dimensionalmente e sem formação de tensões internas. $O$ fator anisotrópico comumente varia de 1,5 a 2,5, e tem se tornado um índice relevante nos estudos de contração de madeira; quanto maior for essa relação, maior será a propensão ao fendilhamento e empenamento da madeira. Para usos que envolverem estabilidade dimensional da madeira, a mais recomendada é aquela que apresentar a menor taxa T/R (OLIVEIRA E SILVA, 2003; SCANAVACA JÚNIOR E GARCIA, 2004; KLITZKE, 2007). Diante do exposto, a caracterização das propriedades físicas de madeiras tropicais comercializadas pelo setor de base florestal de Mato Grosso é expressiva contribuição científica em geral. 


\section{PROTOCOLO EXPERIMENTAL}

Esta pesquisa foi desenvolvida no Laboratório de Tecnologia da Madeira (LTM) do Departamento de Engenharia Florestal (EFL) da Universidade de Brasília (UnB), no estado de Goiás - GO.

\section{Madeiras Estudadas}

As espécies caracterizadas neste estudo foram: Cambará (Qualea brevipedicellata Stafleu), família Vochysiaceae; Cupiúba (Goupia glabra Aubl.), família Goupiaceae; Itaúba (Mezilaurus itauba (Meisn.) Taub. ex Mez), famíla Lauraceae; Cedrinho (Erisma uncinatum Warm.), família Vochysiaceae; Angelim Pedra (Hymenolobium petraeum Ducke), família Fabaceae; Maçaranduba (Manilkara huberi (Ducke) A. Chev.), família Sapotaceae; Garapeira (Apuleia leiocarpa (Vogel) J. F. Macbr), família Fabaceae; Cumarú (Dipteryx odorata (Aubl.) Willd.), família Fabaceae; Marupá (Simarouba amara Aubl.), família Simaroubaceae; Cedrorana (Cedrelinga cateniformis (Ducke) Ducke), família Fabaceae; Tauari (Couratari oblongifolia Ducke \& Kunth), família Lecythidaceae; Jatobá (Hymenaea courbaril L.), família Fabaceae; Angelim-vermelho (Dinizia excelsa Ducke), família Fabaceae; Ipê (Handroanthus serratifolius (A. H. Gentry) S.Grose), família Bignoniaceae; Abiurana (Pouteria egregia Sandwith), família Sapotaceae; Muiracatiara (Tetragastris altissima (Aubl.) Swart.), família Burseraceae; Cambará rosinha (Vochysia máxima Ducke), família Vochysiaceae; Sucupira (Diplotropis purpurea (Rich.) Amshoff.), família Fabaceae; Louro faia (Euplassa pinnata (Lam.) I. M. Johnst.), família Proteaceae e Embira sapo (Lonchocarpus cultratus (Vell.) A. M. G. Azevedo \& H. C. Lima), família Fabaceae.

\section{Corpos de prova}

Para cada espécie foram utilizadas de três a cinco pranchas doadas pelo Instituto de Metrologia e Qualidade do Estado de Mato Grosso, localizado no distrito industrial de Cuiabá-MT/Brasil. A partir das pranchas, foram desdobrados 20 corpos de prova de cada espécie, orientados nas seções longitudinal, radial e tangencial com dimensões de $2 \mathrm{~cm} \times 2 \mathrm{~cm} \times 10 \mathrm{~cm}$, estas dimensões seguem a norma COPANT (1972a). 
Os corpos de prova foram acondicionados em câmara de climatização com temperatura e umidade controlada ( $\pm 65 \%$ UR, $\pm 21^{\circ} \mathrm{C}$ ) para estabilização do teor de umidade ( $12 \%$ de umidade), de acordo com a norma COPANT (1972b).

\section{Densidade}

A metodologia utilizada para a determinação da densidade básica seguiu a norma COPANT (1972c), em que os corpos de prova foram submersos em água por quarenta dias para obtenção volume saturado pelo método estequiométrico utilizando um paquímetro digital, em seguida, acondicionados em estufa a uma temperatura de $103 \pm 2^{\circ} \mathrm{C}$ até atingirem massa constante para obtenção da massa seca ( $0 \%$ umidade) com a utilização da balança digital, com precisão de $0,01 \mathrm{~g}$. A densidade básica foi determinada utilizando a Equação (1):

$$
D B \frac{M \text { massa seca }}{V \text { volume saturado }}
$$

Em que: $\mathrm{DB}=$ Densidade Básica $\left(\mathrm{g} / \mathrm{cm}^{3}\right) ; \mathrm{M}=$ Massa seca $(\mathrm{g}) ;$ Vsaturado = Volume saturado $\left(\mathrm{cm}^{3}\right)$.

\section{Variação dimensional}

A metodologia utilizada para determinação da retratibilidade seguiu a norma COPANT (1972d), onde foram mensuradas as faces longitudinais, radiais e tangenciais dos corpos de prova com um paquímetro digital, nas condições saturados e secos em estufa a uma temperatura de $103 \pm 2^{\circ} \mathrm{C}(0 \%$ de umidade). Os mesmos corpos de prova utilizados para determinação da densidade serão utilizados para estimar a retratibilidade.

Foram efetuadas três medições na face radial, três na face tangencial e uma na longitudinal totalizando sete medições na condição saturada e sete na condição seca em estufa por corpo de prova e realizada a média.

As retratibilidades radial, tangencial e volumétrica foram determinadas utilizando as Equações 2, 3 e 4, respectivamente. 0 coeficiente de anisotropia foi determinado pela Equação 5. 


$$
R R \frac{\text { Dr saturada-Dr seca }}{\text { Dr saturada }} \times 100
$$

Em que: $R R$ = Retratibilidade radial (\%); Dr saturada = dimensão do corpo de prova na direção radial, em condição saturada $(\mathrm{mm})$; Dr seca = dimensão do corpo de prova na direção radial, em condição seca $(\mathrm{mm})$;

$$
R T \frac{\text { Dt saturada-Dt seca }}{\text { Dt saturada }} \times 100
$$

Em que: RT = Retratibilidade tangencial (\%); Dt saturada = dimensão do corpo de prova na direção tangencial, em condição saturada $(\mathrm{mm})$; Dt seca = dimensão do corpo de prova na direção tangencial, em condição seca $(\mathrm{mm})$;

$$
R V \frac{\text { Dv saturado-Dv seco }}{\text { Dv saturado }} \times 100
$$

Em que: RV = Retratibilidade volumétrica (\%); Vsaturado = volume do corpo de prova na condição saturada $\left(\mathrm{cm}^{3}\right) ;$ Vseco = volume do corpo de prova na condição seca $\left(\mathrm{cm}^{3}\right)$;

$$
C A \frac{R T}{R R}
$$

Em que: $\mathrm{CA}=$ Coeficiente de anisotropia; $\mathrm{RT}=$ Retratibilidade tangencial (\%); RR = Retratibilidade radial (\%);

\section{Análise Estatística}

A análise estatística empregada na caracterização das propriedades físicas foi a descritiva: média, máximo, mínimo, desvio padrão e coeficiente de variação. As correlações foram determinadas pelo método de correlação de Pearson a 1\% e 5 \% de significância pelo teste t. O programa estatístico Genes da UFV foi utilizado na análise dos dados (CRUZ, 2006).

\section{RESULTADOS E DISCUSSÕES}

\section{Densidade Básica e Retratibilidade}

A estatística descritiva para a densidade básica, retratibilidades e coeficiente de anisotropia das vinte espécies analisadas são apresentadas na Tabela 1. A densidade básica média apresentou valores oscilando entre $0,33 \mathrm{~g} / \mathrm{cm}^{3}$ a $0,94 \mathrm{~g} / \mathrm{cm}^{3}$, com média geral 
para todas as espécies de $0,64 \mathrm{~g} / \mathrm{cm}^{3}$. O coeficiente de variação (\%) foi baixo e ficou entre $1,75 \%$ e $7,06 \%$, o que implica em uma maior precisão do experimento. Estas características são importantíssimas sob a ótica comercial, pois agregam valor, uso, qualidade e uniformidade aos produtos finais.

\begin{tabular}{|c|c|c|c|c|c|}
\hline \multirow{2}{*}{ ESPÉCIE } & \multirow{2}{*}{$\begin{array}{c}\text { Densidade } \\
\text { Básica } \\
\left(\mathrm{g} / \mathrm{cm}^{3}\right)\end{array}$} & \multicolumn{3}{|c|}{ Retratibibilidade (\%) } & \multirow{2}{*}{$\begin{array}{c}\text { Anisotropia } \\
\text { Razão T/R }\end{array}$} \\
\hline & & Radial & Tangencial & Volumétrica & \\
\hline & 0,59 & 5,46 & 7,42 & 12,85 & 1,38 \\
\hline Q. brevipedicellata & $\begin{array}{l}(0,56) \\
(0,67) \\
(4,01) \\
\end{array}$ & $\begin{array}{c}(4,24) \\
(6,93) \\
(15,06)\end{array}$ & $\begin{array}{c}(5,38) \\
(9,53) \\
(13,58) \\
\end{array}$ & $\begin{array}{l}(10,64) \\
(16,54) \\
(10,77)\end{array}$ & $\begin{array}{c}(1,05) \\
(2,03) \\
(20,01)\end{array}$ \\
\hline & 0,65 & 5,35 & 7,96 & 13,05 & 1,55 \\
\hline G. glabra & $\begin{array}{l}(0,62) \\
(0,68) \\
(2,73) \\
\end{array}$ & $\begin{array}{c}(3,80) \\
(8,09) \\
(23,41) \\
\end{array}$ & $\begin{array}{c}(6,69) \\
(9,88) \\
(10,05)\end{array}$ & $\begin{array}{l}(11,37) \\
(16,45) \\
(11,19) \\
\end{array}$ & $\begin{array}{c}(1,04) \\
(2,33) \\
(21,78) \\
\end{array}$ \\
\hline & 0,67 & 3,2 & 5,62 & 9,23 & 1,86 \\
\hline M. itauba & $\begin{array}{l}(0,63) \\
(0,70) \\
(2,31) \\
\end{array}$ & $\begin{array}{c}(1,96) \\
(4,87) \\
(25,13) \\
\end{array}$ & $\begin{array}{c}(4,28) \\
(7,96) \\
(17,68) \\
\end{array}$ & $\begin{array}{c}(6,85) \\
(12,98) \\
(13,51) \\
\end{array}$ & $\begin{array}{c}(1,04) \\
(3,36) \\
(29,91) \\
\end{array}$ \\
\hline E. uncinatum & $\begin{array}{c}0,47 \\
(0,42) \\
(0,53) \\
(5,59) \\
\end{array}$ & $\begin{array}{c}5,31 \\
(2,53) \\
(7,84) \\
(27,20)\end{array}$ & $\begin{array}{c}7,57 \\
(3,87) \\
(12,69) \\
(25,03) \\
\end{array}$ & $\begin{array}{c}12,85 \\
(7,71) \\
(19,10) \\
(21,32) \\
\end{array}$ & $\begin{array}{c}1,48 \\
(1,00) \\
(2,55) \\
(26,04) \\
\end{array}$ \\
\hline H. petraeum & $\begin{array}{c}0,5 \\
(0,43) \\
(0,56) \\
(7,06) \\
\end{array}$ & $\begin{array}{c}3,6 \\
(2,42) \\
(5,38) \\
(18,64) \\
\end{array}$ & $\begin{array}{c}5,52 \\
(4,45) \\
(7,64) \\
(16,25) \\
\end{array}$ & $\begin{array}{c}9,86 \\
(8,12) \\
(12,01) \\
(12,39) \\
\end{array}$ & $\begin{array}{c}1,58 \\
(0,96) \\
(2,50) \\
(25,65) \\
\end{array}$ \\
\hline M. huberi & $\begin{array}{c}0,82 \\
(0,80) \\
(0,84) \\
(1,75)\end{array}$ & $\begin{array}{c}6,72 \\
(4,79) \\
(9,57) \\
(19,67)\end{array}$ & $\begin{array}{c}10,22 \\
(8,78) \\
(11,82) \\
(7,83)\end{array}$ & $\begin{array}{c}16,76 \\
(13,89) \\
(18,95) \\
(8,38)\end{array}$ & $\begin{array}{c}1,58 \\
(1,02) \\
(2,31) \\
(20,20)\end{array}$ \\
\hline A. leiocarpa & $\begin{array}{c}0,64 \\
(0,59) \\
(0,68) \\
(4,66)\end{array}$ & $\begin{array}{c}4,59 \\
(3,33) \\
(5,89) \\
(18,07)\end{array}$ & $\begin{array}{c}7,62 \\
(7,02) \\
(8,13) \\
(4,40)\end{array}$ & $\begin{array}{c}12,32 \\
(10,82) \\
(13,45) \\
(6,84)\end{array}$ & $\begin{array}{c}1,71 \\
(1,22) \\
(2,32) \\
(18,91)\end{array}$ \\
\hline
\end{tabular}




\begin{tabular}{|c|c|c|c|c|c|}
\hline & 0,84 & 4,68 & 6,84 & 11,67 & 1,5 \\
\hline D. odorata & $\begin{array}{l}(0,78) \\
(0,91) \\
(4,42)\end{array}$ & $\begin{array}{c}(3,26) \\
(5,92) \\
(15,94)\end{array}$ & $\begin{array}{c}(5,82)(7,6) \\
(7,35)\end{array}$ & $\begin{array}{c}(9,95) \\
(13,57) \\
(8,65)\end{array}$ & $\begin{array}{c}(1,08) \\
(1,98) \\
(17,31)\end{array}$ \\
\hline & 0,33 & 3,6 & 5,96 & 9,88 & 1,73 \\
\hline S. amara & $\begin{array}{l}(0,31) \\
(0,35) \\
(2,82)\end{array}$ & $\begin{array}{c}(2,43) \\
(5,05) \\
(20,88)\end{array}$ & $\begin{array}{c}(4,69) \\
(7,62) \\
(12,52)\end{array}$ & $\begin{array}{c}(8,42) \\
(12,33) \\
(11,20)\end{array}$ & $\begin{array}{c}(1,00) \\
(2,42) \\
(24,32)\end{array}$ \\
\hline & 0,51 & 4,63 & 6 & 10,84 & 1,33 \\
\hline C. cateniformis & $\begin{array}{l}(0,46) \\
(0,55) \\
(4,84) \\
\end{array}$ & $\begin{array}{c}(2,1)(7,48) \\
(25,99)\end{array}$ & $\begin{array}{c}(2,18) \\
(8,26) \\
(24,03) \\
\end{array}$ & $\begin{array}{c}(4,86) \\
(15,22) \\
(20,56) \\
\end{array}$ & $\begin{array}{c}(0,99) \\
(2,33) \\
(25,30) \\
\end{array}$ \\
\hline & 0,51 & 3,84 & 5,55 & 10,01 & 1,49 \\
\hline C. oblongifolia & $\begin{array}{l}(0,49) \\
(0,55) \\
(3,11) \\
\end{array}$ & $\begin{array}{c}(2,92) \\
(4,88) \\
(15,31)\end{array}$ & $\begin{array}{l}(4,63) \\
(6,56) \\
(9,56) \\
\end{array}$ & $\begin{array}{c}(8,25) \\
(11,88) \\
(9,83) \\
\end{array}$ & $\begin{array}{c}(1,03) \\
(2,08) \\
(21,82) \\
\end{array}$ \\
\hline & 0,8 & 3,7 & 6,38 & 10,42 & 1,78 \\
\hline H. courbaril & $\begin{array}{l}(0,74) \\
(0,85) \\
(4,06) \\
\end{array}$ & $\begin{array}{c}(2,97) \\
(5,54) \\
(17,39)\end{array}$ & $\begin{array}{c}(2,97) \\
(5,54) \\
(17,39)\end{array}$ & $\begin{array}{c}(7,99) \\
(12,61) \\
(10,91) \\
\end{array}$ & $\begin{array}{c}(1,11) \\
(2,42) \\
(20,95) \\
\end{array}$ \\
\hline D. excelsa & $\begin{array}{c}0,82 \\
(0,75) \\
(0,89) \\
(4,21) \\
\end{array}$ & $\begin{array}{c}5,29 \\
(4,30) \\
(7,92) \\
(16,85) \\
\end{array}$ & $\begin{array}{c}7,2 \\
(4,84) \\
(10,77) \\
(19,22)\end{array}$ & $\begin{array}{c}13,06 \\
(10,14) \\
(17,01) \\
(14,84) \\
\end{array}$ & $\begin{array}{c}1,38 \\
(1,08) \\
(2,04) \\
(6,39) \\
\end{array}$ \\
\hline H. serratifolius & $\begin{array}{c}0,94 \\
(0,86) \\
(1,01) \\
(4,09)\end{array}$ & $\begin{array}{c}6,69 \\
(4,15) \\
(8,20) \\
(14,37)\end{array}$ & $\begin{array}{c}7,48 \\
(4,29) \\
(9,97) \\
(16,23)\end{array}$ & $\begin{array}{c}14,61 \\
(9,69) \\
(18,41) \\
(12,63)\end{array}$ & $\begin{array}{c}1,12 \\
(1,02) \\
(1,25) \\
(18,23)\end{array}$ \\
\hline P. egregia & $\begin{array}{c}0,67 \\
(0,64) \\
(0,71) \\
(2,41) \\
\end{array}$ & $\begin{array}{c}5,83 \\
(4,63) \\
(7,33) \\
(16,21) \\
\end{array}$ & $\begin{array}{c}8,43 \\
(6,03) \\
(11,24) \\
(15,29) \\
\end{array}$ & $\begin{array}{c}14,18 \\
(11,98) \\
(18,51) \\
(10,35) \\
\end{array}$ & $\begin{array}{c}1,49 \\
(1,01) \\
(1,88) \\
(22,58) \\
\end{array}$ \\
\hline T. altissima & $\begin{array}{c}0,67 \\
(0,63) \\
(0,70) \\
(3,11) \\
\end{array}$ & $\begin{array}{c}4,63 \\
(3,27) \\
(8,41) \\
(28,91)\end{array}$ & $\begin{array}{c}8,23 \\
(5,48) \\
(11,79) \\
(26,35)\end{array}$ & $\begin{array}{c}12,09 \\
(8,65) \\
(16,52) \\
(17,14) \\
\end{array}$ & $\begin{array}{c}1,86 \\
(1,06) \\
(2,98) \\
(29,68) \\
\end{array}$ \\
\hline V. máxima & $\begin{array}{c}0,48 \\
(0,45) \\
(0,51) \\
(3,17) \\
\end{array}$ & $\begin{array}{c}5,01 \\
(1,65) \\
(6,71) \\
(27,57) \\
\end{array}$ & $\begin{array}{c}7,48 \\
(2,66) \\
(10,98) \\
(31,58)\end{array}$ & $\begin{array}{c}12,49 \\
(4,81) \\
(18,13) \\
(28,3) \\
\end{array}$ & $\begin{array}{c}1,5 \\
(1,01) \\
(1,96) \\
(19,88) \\
\end{array}$ \\
\hline
\end{tabular}




\begin{tabular}{cccccc}
\hline & 0,77 & 5,18 & 7,11 & 12,46 & 1,43 \\
\cline { 2 - 6 } D. purpurea & $(0,68)$ & $(2,18)$ & $(4,00)$ & $(7,26)$ & $(1,00)$ \\
& $(0,82)$ & $(8,81)$ & $(11,41)$ & $(19,36)$ & $(2,19)$ \\
& $(3,88)$ & $(27,64)$ & $(19,98)$ & $(18,95)$ & $(20,79)$ \\
\hline \multirow{5}{*}{ E. pinnata } & 0,49 & 7,51 & 11,63 & 18,43 & 1,59 \\
\cline { 2 - 6 } & $(0,44)$ & $(5,34)$ & $(6,99)$ & $(13,71)$ & $(1,00)$ \\
& $(0,54)$ & $(11,13)$ & $(14,83)$ & $(21,63)$ & $(2,66)$ \\
& $(5,8)$ & $(17,74)$ & $(21,32)$ & $(13,66)$ & $(27,23)$ \\
\hline \multirow{3}{*}{ L. cultratus } & 0,49 & 4,2 & 6,47 & 11,87 & 1,59 \\
\cline { 2 - 6 } & $(0,43)$ & $(2,70)$ & $(5,28)$ & $(9,96)$ & $(1,09)$ \\
& $(0,53)$ & $(5,17)$ & $(8,38)$ & $(19,65)$ & $(2,56)$ \\
& $(5,48)$ & $(18,79)$ & $(14,01)$ & $(18,24)$ & $(23,48)$ \\
\hline
\end{tabular}

Tabela 1 - Valores médios, mínimos, máximos e coeficientes variação da densidade básica, das retratibilidades e dos coeficientes de anisotropia das espécies estudadas

*Valores entre parênteses são, respectivamente, valores mínimos, máximos e coeficiente de variação (\%).

Silva Filho et al. (1992) afirmam a existência de grandes diferenças na densidade básica de espécies nativas, observando também que a madeira de certas áreas apresenta maior rendimento que a madeira da mesma espécie encontrada em outros locais.

Confirmando os resultados, Araújo (2002) que utilizou fontes institucionais como LPF/IBAMA, IPT, INPA e SUDAM, analisando as características físicas e mecânicas de cento e oitenta e sete espécies nativas da floresta Amazônica, encontrou densidade básica média de $0,63 \mathrm{~g} / \mathrm{cm}^{3}$, com valores mínimos e máximos oscilando de 0,29 $\mathrm{g} / \mathrm{cm}$ a $1,01 \mathrm{~g} / \mathrm{cm}^{3}$.

A densidade básica é uma das propriedades físicas mais importantes de um material, porque está diretamente associada a propriedades como resistência mecânica da madeira e grau de alteração dimensional e perda ou absorção de água (LOBÃO e PEREIRA, 2005). Lopes e Garcia (2002) afirmam que as variações da densidade básica (DB) entre espécies florestais estão relacionadas também com sua anatomia.

As variações de densidade básica encontradas neste estudo estão em conformidade com os valores encontrados na literatura para todas as espécies (IBDF 1981; IBDF 1988; MARQUES, 1997; ARAÚJO, 2002; FERRAZ et al., 2004; ARAÚJO, 2007) com algumas exceções decorrentes provavelmente da idade da árvore, sítio, 
fatores climáticos e/ou constituintes anatômicos e químicos, que são variáveis de espécie para espécie ou indivíduo para indivíduo. De acordo com Moreschi (2010), a densidade básica apresenta variações entre as espécies, dentro de uma mesma espécie e até dentro de uma mesma árvore, tanto no sentido base-topo, quanto no sentido medula-casca.

Melo et al. (1990) estabeleceu uma classificação para a densidade básica (DB) de madeiras tropicais: madeira leve (DB $\leq 500$ $\mathrm{kg} / \mathrm{m} 3)$ - grupo 1; madeira média $(500 \mathrm{~kg} / \mathrm{m} 3<\mathrm{DB} \leq 720 \mathrm{~kg} / \mathrm{m} 3)$ - grupo 2 e madeira pesada (DB>720kg/m3) - grupo 3.

Conforme essa classificação, madeiras tropicais quanto à densidade básica podem variar de leve a pesada. Observando a Tabela 5.3, 30\% das espécies estudadas pertence ao grupo 1 (leve): $E$. uncinatum, H. petraeum, S. amara, V. máxima, E. pinnata e L. cultratus. A maioria das espécies (40\%) se enquadram no grupo 2 (média densidade): Q. brevipedicellata, G. glabra, M. itauba, A. leiocarpa, C. cateniformis, C. oblongifolia, P. egregia e T. altíssima. As madeiras $M$. huberi, $D$. odorata, $H$. courbaril, $D$. excelsa, $H$. serratifolius e $D$. purpurea que somam os últimos $30 \%$, são consideradas pesadas. Araújo (2007) enfatiza que a densidade das espécies florestais é a propriedade que mais fornece informações sobre as características da madeira, por estar intimamente relacionada com as demais propriedades da madeira.

Um bom indicador da estabilidade dimensional da madeira e dos produtos derivados é a amplitude da retratibilidade (GONÇALEZ et al., 2006). Ainda segundo estes autores, a diferença entre as retratibilidades tangencial e radial, frequentemente, explica as deformações na madeira durante a secagem, sendo um parâmetro essencial na qualidade da madeira e, por consequência, em sua utilização.

A classificação das madeiras quanto à retratibilidade volumétrica pode variar de fraca a forte. Segundo Sallenave (1955) e Guiscafre (1978) citados por Gonçalez (1993), espécies com retratibilidade volumétrica forte apresentam valores de 14,1 $19 \%$, espécies com retratibilidade volumétrica média apresentam valores de 9,1 - 14\% e espécies com retratibilidade volumétrica fraca apresentam valores de $4-9 \%$. Observando a Tabela 1, 80\% das espécies pertencem ao grupo das madeiras com retratibilidade 
volumétrica média. As espécies $M$. huberi, $H$. serratifolius, P. egregia e $E$. pinnata foram classificadas com retratibilidade volumétrica forte. Segundo Gonçalez e Gonçalves (2001), as espécies que possuem retratibilidade volumétrica média devem ser serradas após a umidade das mesmas entrarem em equilíbrio com o ambiente. Enquanto as que possuem retratibilidade forte merecem maiores cuidados, devendo ser preferencialmente secas individualmente.

Para Durlo e Marchiori (1992), o índice mais significativo para se avaliar a estabilidade dimensional da madeira é o coeficiente de anisotropia ou fator anisotrópico, sendo definido pela relação entre as contrações $T / R$ (tangencial e radial), sendo frequentemente a contração na direção tangencial cerca de duas vezes maior que na direção radial. Moreschi (2012) classificou o fator anisotrópico (CA) em: madeira considerada excelente $(1,2-1,5)$; madeira considerada normal $(1,6-1,9)$ e madeira de baixa qualidade $(\geq 2,0)$. Sendo assim, quanto mais próximo de 1 esse coeficiente, melhor será a estabilidade dimensional, produzindo madeira de melhor qualidade.

No presente trabalho, a média geral das espécies analisadas mostrou um coeficiente de anisotropia de 1,5, o que permite classificar a madeira como excelente, de acordo com o critério de avaliação proposto por Moreschi (2012), existindo oscilações entre 1,1 para o ipê ( $H$. serratifolius) classificado como excelente e 1,8 para muiracatiara (T. altíssima) classificada como normal.

Correlação entre a Densidade Básica, Retratibilidades Radial, Tangencial, Volumétrica e coeficiente de anisotropia das espécies em estudo.

As correlações obtidas para as propriedades de densidade básica, retratibilidade radial, tangencial e volumétrica e coeficiente anisotropia das espécies estudadas (Tabela 2).

\begin{tabular}{|c|c|c|c|c|c|c|c|c|c|}
\hline ESPÉCIE & & DB & & RR & & RT & & RV & CA \\
\hline \multirow{5}{*}{$\begin{array}{l}\text { Qualea } \\
\text { brevipedicellata }\end{array}$} & $\mathrm{DB}$ & 1 & & & & & & & \\
\hline & $\mathrm{RR}$ & $-0,563$ & $* *$ & 1 & & & & & \\
\hline & RT & $-0,2368$ & ns & 0,1283 & ns & 1 & & & \\
\hline & $\mathrm{RV}$ & $-0,4657$ & $*$ & 0,6353 & $* *$ & 0,8296 & $* *$ & 1 & \\
\hline & $\mathrm{CA}$ & 0,2665 & ns & $-0,6953$ & $* *$ & 0,6095 & $* *$ & 0,0893 ns & 1 \\
\hline
\end{tabular}

Continua... 


\begin{tabular}{|c|c|c|c|c|c|c|c|c|c|c|}
\hline \multirow{5}{*}{$\begin{array}{c}\text { Goupia } \\
\text { glabra }\end{array}$} & $\mathrm{DB}$ & 1 & & & & & & & & \\
\hline & $\mathrm{RR}$ & 0,1658 & ns & 1 & & & & & & \\
\hline & RT & $-0,139$ & ns & 0,241 & ns & 1 & & & & \\
\hline & $\mathrm{RV}$ & $-0,0532$ & ns & 0,8707 & $* *$ & 0,5154 & $*$ & 1 & & \\
\hline & CA & $-0,2482$ & ns & $-0,8392$ & $* *$ & 0,2872 & ns & $-0,5277$ & $*$ & 1 \\
\hline \multirow{5}{*}{$\begin{array}{l}\text { Mezilaurus } \\
\text { itauba }\end{array}$} & DB & 1 & & & & & & & & \\
\hline & $\mathrm{RR}$ & $-0,2037$ & ns & 1 & & & & & & \\
\hline & RT & $-0,0597$ & ns & 0,0312 & ns & 1 & & & & \\
\hline & $\mathrm{RV}$ & $-0,1658$ & ns & 0,6212 & $* *$ & 0,7864 & $* *$ & 1 & & \\
\hline & $\mathrm{CA}$ & 0,1466 & ns & $-0,7837$ & $* *$ & 0,5535 & $*$ & $-0,041$ & ns & 1 \\
\hline \multirow{5}{*}{$\begin{array}{l}\text { Erisma } \\
\text { uncinatum }\end{array}$} & DB & 1 & & & & & & & & \\
\hline & $\mathrm{RR}$ & 0,0557 & ns & 1 & & & & & & \\
\hline & RT & 0,1399 & ns & 0,596 & $* *$ & 1 & & & & \\
\hline & $\mathrm{RV}$ & 0,1213 & ns & 0,8632 & $* *$ & 0,9079 & $* *$ & 1 & & \\
\hline & CA & 0,0541 & ns & $-0,5345$ & $*$ & 0,3269 & ns & $-0,0594$ & ns & 1 \\
\hline \multirow{5}{*}{$\begin{array}{l}\text { Hymenolobium } \\
\text { petraeum }\end{array}$} & DB & 1 & & & & & & & & \\
\hline & $\mathrm{RR}$ & 0,1701 & ns & 1 & & & & & & \\
\hline & RT & 0,5358 & $*$ & $-0,0187$ & ns & 1 & & & & \\
\hline & $\mathrm{RV}$ & 0,4957 & $*$ & 0,3438 & ns & 0,8495 & $* *$ & 1 & & \\
\hline & CA & 0,1335 & ns & $-0,7199$ & $* *$ & 0,673 & $* *$ & 0,3112 & ns & 1 \\
\hline \multirow{5}{*}{$\begin{array}{l}\text { Manilkara } \\
\text { huberi }\end{array}$} & DB & 1 & & & & & & & & \\
\hline & $\mathrm{RR}$ & $-0,4247$ & ns & 1 & & & & & & \\
\hline & RT & 0,0637 & ns & 0,0091 & ns & 1 & & & & \\
\hline & $\mathrm{RV}$ & $-0,3246$ & ns & 0,8209 & $* *$ & 0,5211 & $*$ & 1 & & \\
\hline & CA & 0,4166 & ns & $-0,9255$ & $* *$ & 0,3095 & ns & $-0,6037$ & $* *$ & 1 \\
\hline \multirow{5}{*}{$\begin{array}{l}\text { Apuleia } \\
\text { leiocarpa }\end{array}$} & $\mathrm{DB}$ & 1 & & & & & & & & \\
\hline & $\mathrm{RR}$ & 0,7519 & $* *$ & 1 & & & & & & \\
\hline & RT & 0,2735 & ns & $-0,0833$ & ns & 1 & & & & \\
\hline & $\mathrm{RV}$ & 0,7863 & $* *$ & 0,8768 & $* *$ & 0,3371 & ns & 1 & & \\
\hline & $\mathrm{CA}$ & $-0,7003$ & $* *$ & $-0,9761$ & $* *$ & 0,2513 & ns & $-0,7796$ & $* *$ & 1 \\
\hline \multirow{5}{*}{$\begin{array}{c}\text { Dipteryx } \\
\text { odorata }\end{array}$} & $\mathrm{DB}$ & 1 & & & & & & & & \\
\hline & $\mathrm{RR}$ & $-0,6472$ & $* *$ & 1 & & & & & & \\
\hline & RT & $-0,202$ & ns & 0,158 & ns & 1 & & & & \\
\hline & $\mathrm{RV}$ & $-0,6287$ & $* *$ & 0,7937 & $* *$ & 0,6069 & $* *$ & 1 & & \\
\hline & CA & 0,5799 & $* *$ & $-0,9026$ & $* *$ & 0,2568 & ns & $-0,519$ & $*$ & 1 \\
\hline
\end{tabular}

Continua... 


\begin{tabular}{|c|c|c|c|c|c|c|c|c|c|c|}
\hline \multirow{5}{*}{$\begin{array}{l}\text { Simarouba } \\
\text { amara }\end{array}$} & DB & 1 & & & & & & & & \\
\hline & $\mathrm{RR}$ & $-0,4809$ & $*$ & 1 & & & & & & \\
\hline & RT & 0,259 & ns & $-0,1322$ & ns & 1 & & & & \\
\hline & $\mathrm{RV}$ & $-0,1264$ & ns & 0,5026 & $*$ & 0,6436 & $* *$ & 1 & & \\
\hline & $\mathrm{CA}$ & 0,44 & $*$ & $-0,889$ & $* *$ & 0,5278 & $*$ & $-0,166$ & ns & 1 \\
\hline \multirow{5}{*}{$\begin{array}{l}\text { Cedrelinga } \\
\text { cateniformis }\end{array}$} & DB & 1 & & & & & & & & \\
\hline & $\mathrm{RR}$ & $-0,4945$ & $*$ & 1 & & & & & & \\
\hline & $\mathrm{RT}$ & $-0,2013$ & ns & 0,6159 & $* *$ & 1 & & & & \\
\hline & $\mathrm{RV}$ & $-0,3413$ & ns & 0,8795 & $* *$ & 0,9076 & $* *$ & 1 & & \\
\hline & $\mathrm{CA}$ & 0,3891 & ns & $-0,3963$ & ns & 0,4486 & $*$ & 0,0653 & ns & 1 \\
\hline \multirow{5}{*}{$\begin{array}{l}\text { Couratari } \\
\text { oblongifolia }\end{array}$} & DB & 1 & & & & & & & & \\
\hline & $\mathrm{RR}$ & 0,2195 & ns & 1 & & & & & & \\
\hline & RT & $-0,1319$ & ns & $-0,5242$ & $*$ & 1 & & & & \\
\hline & $\mathrm{RV}$ & 0,5151 & $*$ & 0,3726 & ns & 0,2235 & ns & 1 & & \\
\hline & $\mathrm{CA}$ & $-0,1987$ & ns & $-0,9011$ & $* *$ & 0,8241 & $* *$ & $-0,1056$ & ns & 1 \\
\hline \multirow{5}{*}{$\begin{array}{l}\text { Hymenaea } \\
\text { courbaril L. }\end{array}$} & $\mathrm{DB}$ & 1 & & & & & & & & \\
\hline & $\mathrm{RR}$ & 0,2557 & ns & 1 & & & & & & \\
\hline & RT & $-0,1925$ & ns & $-0,1595$ & ns & 1 & & & & \\
\hline & $\mathrm{RV}$ & 0,1916 & ns & 0,6736 & $* *$ & 0,4865 & $*$ & 1 & & \\
\hline & CA & $-0,2616$ & ns & $-0,7939$ & $* *$ & 0,7051 & $* *$ & $-0,191$ & ns & 1 \\
\hline \multirow{5}{*}{$\begin{array}{l}\text { Dinizia } \\
\text { excelsa }\end{array}$} & $\mathrm{DB}$ & 1 & & & & & & & & \\
\hline & $\mathrm{RR}$ & $-0,257$ & ns & 1 & & & & & & \\
\hline & RT & $-0,3701$ & ns & 0,539 & $*$ & 1 & & & & \\
\hline & $\mathrm{RV}$ & $-0,1969$ & ns & 0,782 & $* *$ & 0,845 & $* *$ & 1 & & \\
\hline & $\mathrm{CA}$ & $-0,1352$ & ns & $-0,353$ & ns & 0,5863 & $* *$ & 0,1789 & ns & 1 \\
\hline \multirow{5}{*}{$\begin{array}{l}\text { Handroanthus } \\
\text { serratifolius }\end{array}$} & DB & 1 & & & & & & & & \\
\hline & $\mathrm{RR}$ & $-0,6107$ & $* *$ & 1 & & & & & & \\
\hline & RT & $-0,6532$ & $* *$ & 0,9096 & $* *$ & 1 & & & & \\
\hline & $\mathrm{RV}$ & $-0,6273$ & $* *$ & 0,9136 & $* *$ & 0,8958 & $* *$ & 1 & & \\
\hline & $\mathrm{CA}$ & $-0,2884$ & ns & 0,1375 & ns & 0,5331 & $*$ & 0,2759 & ns & 1 \\
\hline \multirow{5}{*}{ Pouteria egregia } & $\mathrm{DB}$ & 1 & & & & & & & & \\
\hline & $\mathrm{RR}$ & 0,2675 & ns & 1 & & & & & & \\
\hline & RT & 0,0686 & ns & $-0,0912$ & ns & 1 & & & & \\
\hline & $\mathrm{RV}$ & 0,2838 & ns & 0,5017 & $*$ & 0,7942 & $* *$ & 1 & & \\
\hline & $\mathrm{CA}$ & $-0,179$ & ns & $-0,7757$ & $* *$ & 0,6912 & $* *$ & 0,132 & ns & 1 \\
\hline
\end{tabular}




\begin{tabular}{|c|c|c|c|c|c|c|c|c|c|c|}
\hline \multirow{5}{*}{$\begin{array}{l}\text { Tetragastris } \\
\text { altissima }\end{array}$} & DB & 1 & & & & & & & & \\
\hline & $\mathrm{RR}$ & 0,02 & ns & 1 & & & & & & \\
\hline & RT & 0,2536 & ns & 0,3333 & ns & 1 & & & & \\
\hline & RV & 0,1744 & ns & 0,7385 & $* *$ & 0,6846 & $* *$ & 1 & & \\
\hline & $\mathrm{CA}$ & 0,2129 & ns & $-0,5355$ & $*$ & 0,6076 & $* *$ & $-0,0362$ & ns & 1 \\
\hline \multirow{5}{*}{$\begin{array}{l}\text { Vochysia } \\
\text { máxima }\end{array}$} & DB & 1 & & & & & & & & \\
\hline & $\mathrm{RR}$ & $-0,0503$ & ns & 1 & & & & & & \\
\hline & $\mathrm{RT}$ & 0,0873 & ns & 0,7865 & $* *$ & 1 & & & & \\
\hline & RV & 0,0732 & ns & 0,9007 & $* *$ & 0,9727 & $* *$ & 1 & & \\
\hline & CA & 0,2438 & ns & $-0,0823$ & ns & 0,5326 & $*$ & 0,3386 & ns & 1 \\
\hline \multirow{5}{*}{$\begin{array}{l}\text { Diplotropis } \\
\text { purpurea }\end{array}$} & DB & 1 & & & & & & & & \\
\hline & $\mathrm{RR}$ & $-0,2576$ & ns & 1 & & & & & & \\
\hline & $\mathrm{RT}$ & $-0,3175$ & ns & 0,7655 & $* *$ & 1 & & & & \\
\hline & RV & $-0,3175$ & ns & 0,9527 & $* *$ & 0,911 & $* *$ & 1 & & \\
\hline & $\mathrm{CA}$ & $-0,0232$ & ns & $-0,7335$ & $* *$ & $-0,1627$ & ns & $-0,5258$ & ns & 1 \\
\hline \multirow{5}{*}{$\begin{array}{l}\text { Euplassa } \\
\text { pinnata }\end{array}$} & DB & 1 & & & & & & & & \\
\hline & $\mathrm{RR}$ & 0,1608 & ns & 1 & & & & & & \\
\hline & $\mathrm{RT}$ & 0,1064 & ns & 0,0948 & ns & 1 & & & & \\
\hline & RV & 0,1666 & ns & 0,498 & $*$ & 0,8446 & $* *$ & 1 & & \\
\hline & $\mathrm{CA}$ & 0,007 & ns & $-0,552$ & $*$ & 0,7502 & $* *$ & 0,3855 & ns & 1 \\
\hline \multirow{5}{*}{$\begin{array}{l}\text { Lonchocarpus } \\
\text { cultratus }\end{array}$} & DB & 1 & & & & & & & & \\
\hline & $\mathrm{RR}$ & 0,745 & $* *$ & 1 & & & & & & \\
\hline & $\mathrm{RT}$ & 0,2453 & ns & 0,109 & ns & 1 & & & & \\
\hline & RV & 0,1454 & ns & $-0,0197$ & ns & 0,7481 & $* *$ & 1 & & \\
\hline & CA & $-0,5343$ & $*$ & $-0,81$ & $* *$ & 0,4719 & $*$ & 0,5106 & $*$ & 1 \\
\hline
\end{tabular}

Tabela 2 - Correlação entre Densidade Básica (DB), Retratibilidades (RR, RT, RV) e Coefiente de Anisotropia (CA) para as madeiras estudadas

** e *: Significativo a 1 e 5\%, respectivamente, pelo teste t; ns: Não significativo

Ao se avaliar a correlação entre a densidade básica e a retratibilidade volumétrica para as vinte espécies estudadas, verificou-se uma fraca correlação e não significativa para as espécies Goupia glabra, Mezilaurus itauba, Erisma uncinatum, Manilkara huberi, Simarouba amara, Cedrelinga cateniformis, Hymenaea courbaril, Dinizia excelsa, Pouteria egregia, Tetragastris altíssima, Vochysia máxima, Diplotropis purpurea, Euplassa pinnata e Lonchocarpus cultratus. Corroborando com os resultados, Rocha (1994) observou resultados semelhantes ao avaliar correlações entre as propriedades físicas da madeira de diferentes espécies tropicais. Araújo (2002) correlacionou as propriedades físicas e mecânicas de 187 
espécies nativas, encontrou correlações fracas entre densidade e retratibilidade radial $(0,443)$ e entre densidade e retratibilidade tangencial $(0,411)$. Melo et al. (2013) correlacionou propriedades físicas e mecânicas de 30 espécies nativas do Rio Grande do Sul, para as propriedades físicas, os coeficientes de retratibilidade foram os parâmetros que tiveram as menores correlações que, apesar fracas foram significativas.

As espécies Qualea brevipedicellata, Apuleia leiocarpa, Dipteryx odorata e Handroanthus serratifolius mostraram correlações significativas entre a densidade e as retratibilidades radial e volumétrica. Este comportamento com correlações positivas entre as retratibilidades com a densidade foi verificado por Zerbini (2008) para as espécies nativas Guajará e Taxi e Calderon (2012) para $A$. macrocarpon, S. globulifera, O. costulata e B. paraense.

As espécies Apuleia leiocarpa $(-0,7003)$ e Lonchocarpus cultratus $(-0,5343)$ apresentaram correlação significativa entre densidade básica (DB) e coeficiente de anisotropia (CA), porém negativa. Gonçalves et al. (2010) avaliaram correlações entre as propriedades tecnológicas em um híbrido clonal de Eucalyptus urophylla $x$ Eucalyptus grandis e a densidade básica (DB) apresentou forte correlação negativa com o fator anisotrópico (CA) $(-0,83)$.

Apesar da maioria das espécies estudadas não terem apresentado correlação significativa entre a densidade e as retratibilidades, algumas espécies mostram correlação com algumas das formas de retratibilidade. Observou-se que não existe uma tendência geral de correlação da densidade com as retratibilidades, devendo sempre ser considerado espécie a espécie para poder entender o comportamento das madeiras com relação a estas propriedades. Provavelmente, a constituição anatômica de cada espécie aliada a sua constituição química tem influência nessas propriedades, fazendo com que exista essa variação. Além disso, segundo Gonçalez (1993), o número de amostras, aliado ao não controle dos locais das pranchas de onde foram retirados os corpos de prova também pode ter influenciado os resultados.

Como esperado, houve uma tendência para a maioria das espécies de uma correlação significativa entre as retratibilidades, principalmente da volumétrica com a radial e a tangencial. Segundo Oliveira e Silva (2003), a magnitude das variações dimensionais 
depende de inúmeros fatores, como o teor de umidade, a direção estrutural (radial, tangencial ou longitudinal), a posição dentro da árvore, a densidade da madeira, a temperatura, o grau de estresse de secagem causado pelo gradiente de umidade, entre outros. Os resultados encontrados neste trabalho são similares aos de Gonçalez (1993), Araújo (2002), Zerbini (2008), D’ Ambros (2011) e Calderon (2012), que trabalharam com espécies de madeira tropicais.

\section{CONSIDERAÇÕES FINAIS}

- A densidade das espécies variou de leve a pesada, sendo que a maioria ficou no grupo de densidade média, característica desejável para a indústria.

- Quanto ao uso da madeira em relação a estabilidade dimensional é possível inferir que as espécies estudadas não apresentarão maiores problemas como rachaduras, torções e empenamentos, decorrentes de contrações diferenciadas durante a sua secagem e posterior industrialização.

\section{REFERÊNCIAS}

ARAÚJO, H. J. B. Agrupamento das espécies madeireiras ocorrentes em pequenas áreas sob manejo florestal do projeto de colonização Pedro Peixoto (AC) por similaridade das propriedades físicas e mecânicas. Dissertação de mestrado em Recursos Florestais, Escola Superior de Agricultura "Luiz de Queiroz", Universidade de São Paulo. 184p. 2002.

ARAÚJO, H. J. B. Relações funcionais entre propriedades físicas e mecânicas de madeiras tropicais brasileiras. Revista Floresta, v. 37, n. 3, 399-416. 2007.

BAKER, T. R., PHILLIPS, O. L., MALHI, Y., ALMEIDA, S., ARROYO, L., FIORE, A., ERWIN, T., KILLEN, T. J., LAURANCE, S. G., LAURANCE, W. F., LEWIS, S. L., LLOYD, J. Variation in wood density determines spatial patterns in Amazonian forest biomass. Global Change Biology, 10:545-562, 2004.

BATISTA, D. C. Modificação térmica da madeira de Eucalyptus grandis em escala industrial pelo processo brasileiro vap holzSysteme ${ }^{\circledR}$. Tese de Doutorado em Engenharia Florestal, Universidade Federal do Paraná, 338p, 2012.

BATISTA, D. C.; KLITZKE, R. J.; SANTOS, C. V. T. Densidade básica e retratibilidade da madeira de clones de três espécies de Eucalyptus. Revista Ciência Florestal, Santa Maria, v. 20, n. 4, p. 665-674, out.-dez., 2010. 
CALDERON, C. M. A. O segmento moveleiro na região do Alto Juruá - AC: Perfil e uso de tecnologias alternativas para a caracterização das principais espécies madeireiras. Tese de Doutorado em Ciências Florestais, Departamento de Engenharia Florestal, Universidade de Brasília, 174p. 2012.

COMISSION PANAMERICANA DE NORMAS TÉCNICAS - COPANT. Maderas: selección y collectión de muestras. In: COPANT, Caracas-Venezuela, COPANT - 458. 1972a.

COMISSION PANAMERICANA DE NORMAS TÉCNICAS - COPANT. Maderas: acondicionamiento de las maderas destinadas a los ensayos físicos y mecânicos. COPANT, Caracas-Venezuela, COPANT - 459. 1972b.

COMISSION PANAMERICANA DE NORMAS TÉCNICAS-COPANT. “Maderas: método de determinación del peso especifico aparente". COPANT, Caracas-Venezuela, COPANT - 461. 1972c.

COMISSION PANAMERICANADE NORMASTÉCNICAS-COPANT. “Maderas: método de determinación de la contracción”. COPANT, Caracas-Venezuela, COPANT - 462.1972d.

CRUZ, C. D. Programa Genes: Biometria. Editora UFV. Viçosa (MG). 382p. 2006.

D'AMBROS, J. Cadeia produtiva moveleira da região central do Estado do Tocantins: caracterização e perspectivas para a formação de polo moveleiro. Tese de doutorado em Ciências Florestais, Departamento de Engenharia Florestal, Universidade de Brasília-UnB, 301p. 2011.

DURLO, M. A., MARCHIORI, J. N. C. Tecnologia da madeira: Retratibilidade. Santa Maria, CEPEF/FATEC, Série Técnica, 10. 33p. 1992.

FERRAZ, I. D. K., LEAL FILHO, N., IMAKAWA, A. M., VARELA, V. P., PIÑA-RODRIGUES, F. C. P. Características básicas para um agrupamento ecológico preliminar de espécies madeireiras da floresta de terra firme da Amazônia Central. Revista Acta Amazônica, VOL. 34(4): 621 - 633. 2004.

GONÇALEZ, J. C. Caracterisation technologique de quatre espéces peu connues da la forêt Amazonienne: anatomie, chimie, couleur, propriétés physiques et mécaniques. Thèse Doctorat - Ecole Nationale du Génie Rural des Eaux et des Forêts, Nancy, 446p. 1993.

GONÇALEZ, J. C., BREDA, L. C. S., BARROS, J. F. M., MACEDO, D. G., JANIN, G., COSTA, A. F., VALE, A. T. Características tecnológicas das madeiras de Eucalyptus grandis W.Hill ex Madein e Eucalyptus cloeziana F. Muell visando ao seu aproveitamento na indústria moveleira. Revista Ciência Florestal, Santa Maria, v. 16, n. 3, 329-341. 2006.

GONÇALEZ, J. C.; GONÇALVES, D. M. Valorização de duas espécies de madeira Cedrelinga catenaeformis e Enterolobium shomburgkii para a indústria madeireira. Revista Brasil Florestal, № 70, junho de 2001.

GONÇALVES, F. G., OLIVEIRA, J. T. S., SILVA, G. F., NAPPO, M. E., TOMAZELLO FILHO, M. Parâmetros dendrométricos e correlações com propriedades tecnológicas em 
um híbrido clonal de Eucalyptus urophylla x Eucalyptus grandis. Revista Árvore, Viçosa-MG, v.34, n.5, 947-959. 2010.

INSTITUTO BRASILEIRO DE DESENVOLVIMENTO FLORESTAL - IBDF. Madeiras da Amazônia: características e utilização. Volume 1, Floresta Nacional do Tapajós. Laboratório de Produtos Florestais - LPF, IBAMA, 113p. 1981.

INSTITUTO BRASILEIRO DE DESENVOLVIMENTO FLORESTAL - IBDF. Madeiras da Amazônia: características e utilização. Volume 2, estação experimental de Curuá-una. Laboratório de Produtos Florestais - LPF, IBAMA, 235p. 1988.

KLITZKE, R. J. Secagem da madeira. In: OLIVEIRA, J. T. S., FIEDLER, N. C., NOGUEIRA, M. Tecnologias aplicadas ao setor florestal brasileiro. Jerônimo Monteiro. 2007.

LOBÃO, M. S., DELLA LÚCIA, R. M., MOREIRA, M. S. S., GOMES, A. Caracterização das propriedades físico-mecânicas da madeira de eucalipto com diferentes densidades. Revista Árvore, Viçosa-MG, v.28, n.6, 889-894, 2004.

LOBÃO, M. S., PEREIRA, K. R. M. Propriedades Físicas e Mecânicas da Madeira Universidade Federal do Acre, Departamento de Ciências agrárias - Rio Branco/ AC. Série tecnologia da madeira, Apostila, V. 1, n1, 44p. 2005.

LOPES, G. A., GARCIA, J. N. Densidade básica e umidade natural da madeira de Eucalyptus saligna Smith, de Itatinga, associadas aos padrões de casca apresentados pela população. Revista Scientia Forestalis, n.62, 13-23. 2002.

MARQUES, M. H. B. Madeiras da Amazônia características e utilização. Volume 3, Amazônia oriental, Instituto Brasileiro do Meio Ambiente e dos Recursos Naturais Renováveis - IBAMA, Laboratório de Produtos Florestais-LPF. 141p. 1997.

MARQUES, S. S., OLIVEIRA, J. T. S., PAES, J. B., ALVES, E. S., SILVA, A. G., FIEDLER, N. C. Estudo comparativo da massa específica aparente e retratibilidade da madeira de pau-brasil (Caesalpinia echinata LAM.) nativa e de reflorestamento. Revista Árvore, Viçosa-MG, v.36, n.2, 373-380. 2012.

MELO, J. E.; CORADIN, V. T. R.; MENDES, J. C. Classes de densidade para madeiras da Amazônia brasileira. In: Congresso Florestal Brasileiro, 6, Campos do Jordão. Anais. São Paulo: Sociedade Brasileira de Silvicultura, v.3, p.695-699. 1990.

MELO, R. R., ARALDI, D. B., STANGERLIN, D. M., MÜLLER, M. T., GATTO, D. A. Uso das características tecnológicas da madeira para o agrupamento de espécies florestais. Revista Nativa, Sinop, v. 01, n. 01, 01-07. 2013.

MORESCHI, J. C. Apostila de propriedades da madeira. Setor de Ciências agrárias UFPR, Departamento de Engenharia e Tecnologia Florestal, 2010. Link: <http://www.madeira.ufpr.br/publicacoes/propriedadesdamadeira2010.pdf> Acesso em: 22/02/2016.

MORESCHI, J. C. Apostila de propriedades da madeira. Setor de Ciências agrárias UFPR, Departamento de Engenharia e Tecnologia Florestal. $4^{\circ}$ edição, Link: http:// www.madeira.ufpr.br/disciplinasmoreschi/PROPRIEDADES\%20DA\%20MADEIRA. pdf Acesso em: 15/01/2016. 2012. 
OLIVEIRA, J. T. S. Caracterização da madeira de eucalipto para a construção civil. Tese de Doutorado em Engenharia Civil, Escola Politécnica, Universidade de São Paulo, 429p. 1998.

OLIVEIRA, J. T. S.; SILVA, J. C. Variação radial da retratibilidade e densidade básica da madeira de Eucalyptus saligna Sm. Revista Árvore, Viçosa-MG, v.27, n.3, p.381-385, 2003.

OLIVEIRA, J. T. S., TOMAZELLO FILHO, M., FIEDLER, N. C. Avaliação da retratibilidade da madeira de sete espécies de Eucalyptus. Revista Árvore, Viçosa-MG, v.34, n.5, 929-936. 2010.

ROCHA, J. S. A segurança de estruturas de madeira determinada a partir da variabilidade da densidade básica e de propriedades mecânicas de madeiras Amazônicas. Dissertação de Mestrado em Recursos Florestais, Escola Superior de Agricultura "Luiz de Queiroz", Piracicaba, 160 P. 1994.

SCANAVACA JÚNIOR, L.; GARCIA, J. N. Determinação das propriedades físicas e mecânicas da madeira de Eucalyptus urophylla. Revista Scientia forestais, n. 65; p. 120 a 129 , jun. 2004.

SILVA FILHO, D. F., ROCHA, J. S., MOURA, J. B. Influência da densidade na dureza Janka em oito espécies madeireiras da Amazônia central. Revista Acta Amazônica, 22 (2): $275-283.1992$.

ZERBINI, N. J. Madeiras tropicais com potencial comercial da região do rio Xingu (Pará, Brasil): Propriedades tecnológicas e cadeia produtiva. Tese de doutorado em Ciências Florestais, Faculdade de Tecnologia, Universidade de Brasília, 212p. 2008. 